Mon. Not. R. Astron. Soc. 000, 000-000 (0000) Printed 14 May $2019 \quad$ (MN LATEX style file v2.2)

\title{
Black hole mergers from quadruples
}

\author{
Giacomo Fragione $^{1 \star}$ and Bence Kocsis ${ }^{2}$ \\ ${ }^{1}$ Racah Institute for Physics, The Hebrew University, Jerusalem 91904, Israel \\ ${ }^{2}$ Institute of Physics, Eötvös University, Pázmány P. s. 1/A, Budapest, 1117, Hungary
}

14 May 2019

\begin{abstract}
With the hundreds of merging binary black hole $(\mathrm{BH})$ signals expected to be detected by LIGO/Virgo, LISA and other instruments in the next few years, the modeling of astrophysical channels that lead to the formation of compact-object binaries has become of fundamental importance. In this paper, we carry out a systematic statistical study of quadruple BHs consisting of two binaries in orbit around their center of mass, by means of high-precision direct $N$-body simulations including Post-Newtonian (PN) terms up to 2.5PN order. We found that most merging systems have high initial inclinations and the distributions peak at $\sim 90^{\circ}$ as for triples, but with a more prominent broad distribution tail. We show that BHs merging through this channel have a significant eccentricity in the LIGO band, typically much larger than $\mathrm{BHs}$ merging in isolated binaries and in binaries ejected from star clusters, but comparable to that of merging binaries formed via the GW capture scenario in clusters, mergers in hierarchical triples, or $\mathrm{BH}$ binaries orbiting intermediate-mass black holes in star clusters. We show that the merger fraction can be up to $\sim 3-4 \times$ higher for quadruples than for triples. Thus even if the number of quadruples is $20 \%-25 \%$ of the number of triples, the quadruple scenario can represent an important contribution to the events observed by LIGO/VIRGO.
\end{abstract}

Key words: Galaxy: kinematics and dynamics - stars: black holes - stars: kinematics and dynamics - galaxies: star clusters: general

\section{INTRODUCTION}

The LIGO-Virgo collaboration has recently released a catalogue of compact object mergers due to gravitational wave (GW) emission, comprised of ten merging black hole $(\mathrm{BH})$ binaries and one merging neutron (NS) binary (The LIGO Scientific Collaboration \& the Virgo Collaboration 2018). As the detector sensitivity is improved, hundreds of merging binary signals are expected to be detected in the next few years, and the modeling of astrophysical channels that lead to the formation of compact-object binaries has become of fundamental importance.

Several astrophysical channels have been proposed leading to merging compact objects. Possibilities include isolated binary evolution through a common envelope phase (Belczynski et al. 2016; Giacobbo \& Mapelli 2018), envelope expansion and fallback (Tagawa et al. 2018) or chemically homogeneous evolution (Mandel \& de Mink 2016; Marchant et al. 2016), Lidov-Kozai (LK) mergers of binaries in galactic nuclei (Antonini \& Perets 2012; Petrovich \& Antonini 2017; Hamers et al. 2018; Hoang et al. 2018; Fragione et al. 2018; Grishin et al. 2018) and in stellar triple systems (Antonini

\footnotetext{
* E-mail: giacomo.fragione@mail.huji.ac.il
}

et al. 2017; Silsbee \& Tremaine 2017; Arca-Sedda et al. 2018; Liu \& Lai 2018), mergers of wide binaries (Michaely \& Perets 2019), GW capture events in galactic nuclei (O'Leary et al. 2009; Rasskazov \& Kocsis 2019), mergers in active galactic nuclei (AGN) accretion disks (Bartos et al. 2017; Stone et al. 2017), mergers in dark matter halos (Bird et al. 2016; Sasaki et al. 2016), and mergers in star clusters (Portegies Zwart \& McMillan 2000; O'Leary et al. 2006; Askar et al. 2017; Banerjee 2018; Choksi et al. 2018; Fragione \& Kocsis 2018; Rastello et al. 2018; Rodriguez et al. 2018). While typically each model accounts for roughly the same rate $\left(\sim\right.$ few $\mathrm{Gpc}^{-3} \mathrm{yr}^{-1}$, with the possible exception of the isolated binary case which predicts possibly much higher rates), the statistical contribution of different astrophysical channels can be hopefully disentangled using the spin, mass, eccentricity and redshift distributions (see e.g. O'Leary et al. 2016; Gondán et al. 2018; Samsing et al. 2018a; Zevin et al. 2019).

Bound stellar multiples are not rare. In particular, for massive stars which are progenitors of NSs and BHs, observations have shown that more than $\sim 50 \%$ and $\sim 15 \%$ have at least one or two stellar companions, respectively (Duquennoy \& Mayor 1991; Raghavan et al. 2010; Sana et al. 2013; Tokovinin 2014a,b; Dunstall et al. 2015; Sana 
2017; Jiménez-Esteban et al. 2019). Most previous dynamical studies on bound multiples have focused on determining the $\mathrm{BH}$ merger rate from isolated bound triples or triple $\mathrm{BHs}$ in globular clusters (Wen 2003; Antonini et al. 2016, 2017; Silsbee \& Tremaine 2017; Arca-Sedda et al. 2018). Quadruple systems are also observed. Riddle et al. (2015) found $\mathrm{a} \sim 4 \%$ abundance of $2+2$ quadruples. Compared to triple systems, quadruples have six additional degrees of freedom (i.e. the Keplerian elements) and excursions to very high eccentricity can take place over a much larger fraction of the parameter space (Pejcha et al. 2013).

The dynamics of $2+2$ quadruple systems has been under scrutiny to investigate a number of astrophysical phenomena, including orbital synchronization (Seto 2018) and white dwarf-white dwarf mergers (Fang et al. 2017; Hamers 2018). Vokrouhlický (2016) and Breiter \& Vokrouhlický (2018) studied the secular and resonant dynamics of $2+2$ systems, while Arca-Sedda et al. (2018) and Zevin et al. (2019) examined binary-binary scattering events, which may lead to eccentric BH mergers. Recently, Liu \& Lai (2019) have discussed the possibility that the richer dynamics of quadruples can lead to a merger fraction $\sim 10$ times higher than that of triple systems, thus highlighting the relevance of this channel.

In this paper, we study for the first time the dynamical evolution of $2+2$ quadruple BHs by means of direct highprecision $N$-body simulations, including Post-Newtonian (PN) terms up to $2.5 \mathrm{PN}$ order. In our calculations, we consider a power-law mass spectrum for the BHs, different maximum extensions of the quadruple and different distributions of the inner and outer semi-major axes and eccentricities. We quantify how the probability of merger depends on the initial conditions and determine the parameter distribution of merging systems relative to the initial distributions.

The paper is organized as follows. In Section 2, we discuss the relevant timescales of the considered systems. In Section 3, we present our numerical methods to determine the rate of $\mathrm{BH}$ mergers in quadruples, and discuss the parameters of merging systems. Finally, in Section 4, we discuss the implications and draw conclusions.

\section{LIDOV-KOZAI MECHANISM IN QUADRUPLES}

Figure 1 illustrates the system considered in this paper. The system consists of two inner binaries with masses $\left(m_{1}, m_{2}\right)$ and $\left(m_{3}, m_{4}\right)$, respectively, whose center of masses orbit around the 4-body system's center of mass, denoted as the outer binary. The semimajor axes and eccentricities of the inner binaries are denoted by $a_{12}, e_{12}$ and $a_{34}, e_{34}$, respectively, and of the outer orbit by $a_{\text {out }}$ and $e_{\text {out }}$.

In the case of a triple system made up of an inner binary that is orbited by a companion, the inner eccentricity and inclination oscillate due to the quadrupole moment of the tidal potential of the third body via the LK mechanism whenever the initial mutual orbital inclination is between $i_{0} \sim 40^{\circ}-140^{\circ}$ (Lidov 1962; Kozai 1962). The semimajor axis is approximately fixed during these oscillation cycles. At the secular quadrupole order of approximation, these os-

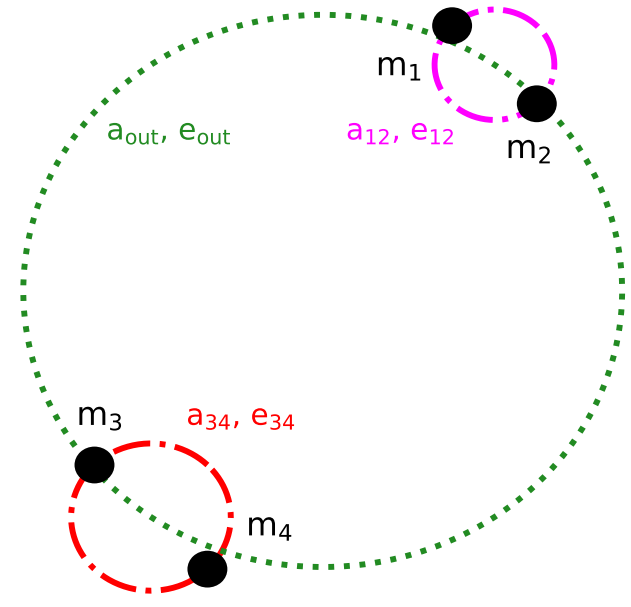

Figure 1. The four-body system studied in the present work. We denote the mass of the components of the first inner binary as $m_{1}$ and $m_{1}$ and of the second inner binary as $m_{3}$ and $m_{4}$. The semimajor axis and eccentricity of the inner binaries are $a_{12}, e_{12}$ and $a_{34}, e_{34}$, respectively, and of the outer orbit $a_{\text {out }}$ and $e_{\text {out }}$.

cillations occur on a timescale (Naoz 2016)

$$
T_{\mathrm{LK}}=\frac{8}{15 \pi} \frac{m_{\mathrm{tot}}}{m_{3 \mathrm{~b}}} \frac{P_{3 \mathrm{~b}}^{2}}{P_{\mathrm{in}}}\left(1-e_{3 \mathrm{~b}}^{2}\right)^{3 / 2},
$$

where $P_{\text {in }}$ and $P_{3 \mathrm{~b}}$ are the orbital periods of the inner and outer binary, respectively, $m_{3 \mathrm{~b}}$ is the mass of the perturber, and $m_{\text {tot }}$ is the total mass of the triple system. At the quadruple order of approximation, the maximal eccentricity of the inner binary due to the LK mechanism depends on the initial mutual inclination

$$
e_{\mathrm{in}}^{\max }=\sqrt{1-\frac{5}{3} \cos ^{2} i_{0}} .
$$

As $i_{0}$ approaches $\sim 90^{\circ}$, the inner binary eccentricity approaches almost unity. In the case of a compact-object binary, the large eccentricity makes its GW merger time shorter since it dissipates energy more efficiently at the pericentre (e.g., see Antognini 2015; Naoz 2016). Nevertheless, LK cycles can be suppressed by additional sources of precession, such as tidal bulges or general relativistic precession, which operate on a typical timescale of (Naoz 2016)

$$
T_{\mathrm{GR}}=\frac{a_{\mathrm{in}}^{5 / 2} c^{2}\left(1-e_{\mathrm{in}}^{2}\right)}{3 G^{3 / 2}\left(m_{\mathrm{in}, 1}+m_{\mathrm{in}, 2}\right)^{3 / 2}} .
$$

If $T_{\mathrm{GR}}<T_{\mathrm{LK}}$, the LK oscillations of the $\left(e_{\mathrm{in}}, i\right)$ orbital elements are damped by relativistic effects (Naoz et al. 2013).

If the octupole corrections are taken into account, the eccentricity excitation becomes more prominent. In the case the outer orbit is eccentric, the inner eccentricity can reach almost unity even if the initial inclination is outside of the $i_{0} \sim 40^{\circ}-140^{\circ}$ Kozai-Lidov range (Naoz et al. 2013; Li et al. 2014).

In the case the third companion is itself a binary star, as assumed in this paper, each binary acts as a distant perturber inducing LK cycles on the other binary. The evolution of such $2+2$ quadruple systems differs from a combination of two uncoupled three-body LK processes. Pejcha et al. (2013) 
Table 1. Models: name, maximum outer semi-major axis $\left(a_{\text {out }}^{\max }\right)$, slope of the $\mathrm{BH}$ mass function $(\beta)$, semi-major axis distribution $(f(a))$, eccentricity distribution $(f(e))$, merger fraction $\left(f_{\mathrm{mer}}\right)$.

\begin{tabular}{lccccc}
\hline Name & $a_{\text {out }}^{\max }(\mathrm{AU})$ & $\beta$ & $f(a)$ & $f(e)$ & $f_{\text {mer }}$ \\
\hline \hline A1 & 1000 & 1 & uniform & uniform & 0.29 \\
A2 & 1000 & 2 & uniform & uniform & 0.29 \\
A3 & 1000 & 3 & uniform & uniform & 0.25 \\
A4 & 1000 & 4 & uniform & uniform & 0.24 \\
B1 & 3000 & 1 & uniform & uniform & 0.26 \\
B2 & 1000 & 1 & log-uniform & uniform & 0.28 \\
B3 & 1000 & 1 & uniform & thermal & 0.35 \\
\hline
\end{tabular}

showed that the binaries can experience coherent eccentricity oscillations and excursions to very high eccentricity that take place over a much larger fraction of the parameter space compared to triple systems. Hamers \& Lai (2017) showed that the reason for this behaviour is that the second binary can induce nodal precession to the outer $(2+2)$ orbit, which can cause a secular resonance if it happens on a timescale comparable to the LK timescale, and make the first binary merge. With respect to the first inner binary, the strength of this effect is parametrised by the dimensionless parameter (Hamers \& Lai 2017)

$$
\eta=\frac{3}{4} \cos I_{2}\left(\frac{a_{34}}{a_{12}}\right)^{3 / 2}\left(\frac{m_{1}+m_{2}}{m_{3}+m_{4}}\right)^{3 / 2},
$$

where $\cos I_{2}$ is the inclination of the orbital plane of the second inner binary compared to the outer orbital plane. When $\eta \sim 1$, the timescale of the induced nodal precession and of the LK mechanism of the first binary are comparable and the resonance has its maximum effect.

Liu \& Lai (2019) have recently discussed that dynamically induced $\mathrm{BH}$ mergers in quadruple systems may be an important channel of producing $\mathrm{BH}$ mergers observed by LIGO/VIRGO due to the richer dynamics of $2+2$ systems.

\section{N-BODY SIMULATIONS: BLACK HOLE MERGERS}

The $\mathrm{BH}$ quadruples in our simulations are initialized as follows. In total, we consider seven different models with different distributions of quadruple parameters (see Tab. 1) to explore the role of the relevant quadruple parameters.

In our models, we sample the masses of the BHs from

$$
\frac{d N}{d m} \propto M^{-\beta}
$$

in the mass range $5 \mathrm{M}_{\odot}-100 \mathrm{M}_{\odot}$ (Hoang et al. 2018). To check how the results depend on the slope of the $\mathrm{BH}$ mass function, we run models with $\beta=1,2,3,4$ (O'Leary et al. $2016)$. The inner $\left(a_{12}, a_{34}\right)$ and outer $\left(a_{\text {out }}\right)$ semi-major axes are drawn either from a uniform distribution $(f(a) \propto$ const $)$ or a $\log$-uniform $(f(a) \propto 1 / a)$ distribution. The minimum inner semimajor axis is $1 \mathrm{AU}$. We also consider two different maximum outer semimajor axis $a_{\max }^{\text {out }}$ for the quadruples (1000 AU or $3000 \mathrm{AU})$. The inner $\left(e_{12}, e_{34}\right)$ and outer $\left(e_{\mathrm{out}}\right)$ eccentricities are sampled from a uniform $(f(e) \propto$ const $)$ or thermal $(f(e) \propto e)$ distribution. The initial mutual inclination $i_{0}$ between the inner and outer orbit is sampled from

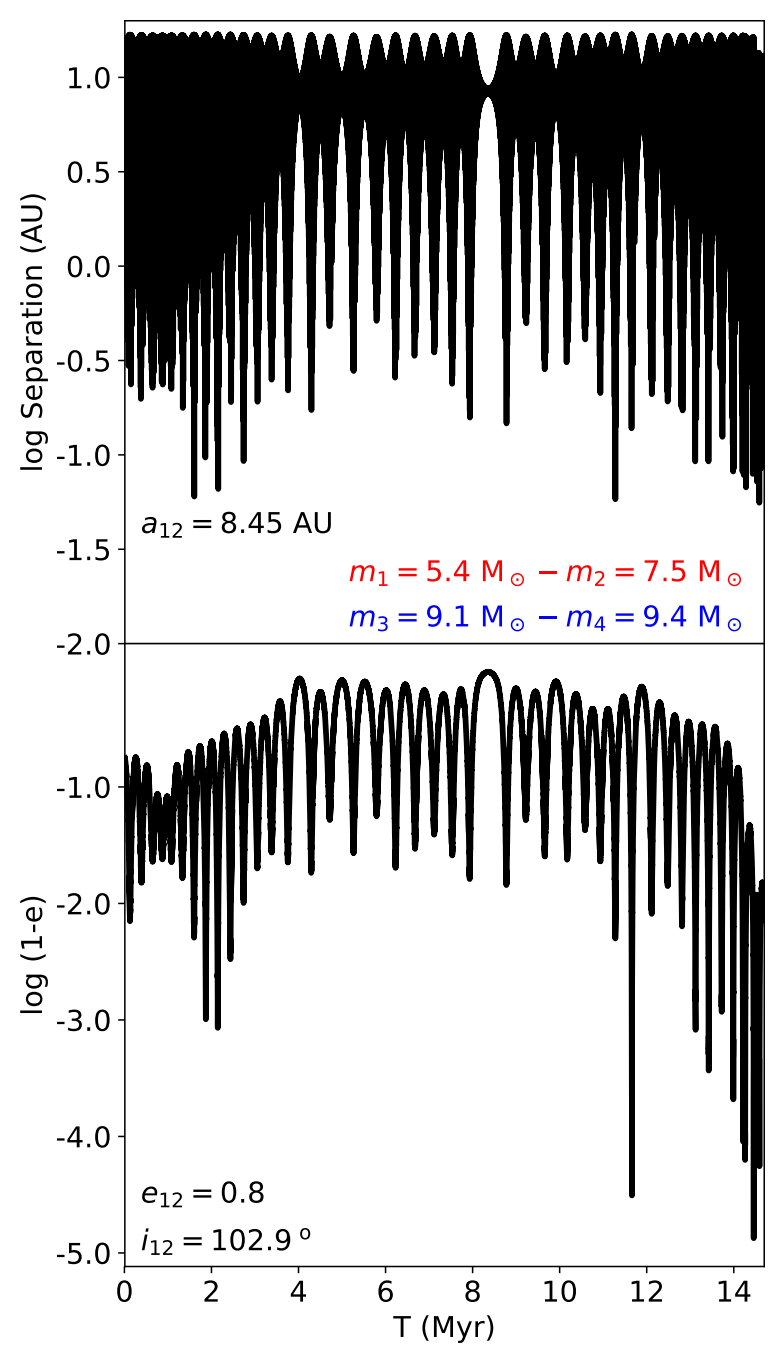

Figure 2. Example of a four-body integration of one quadruple system with masses $m_{1}=5.4 \mathrm{M}_{\odot}, m_{2}=7.5 \mathrm{M}_{\odot}, m_{3}=9.1 \mathrm{M}_{\odot}$, $m_{4}=9.4 \mathrm{M}_{\odot}$. The first two BHs start with a semi-major axis $a_{12}=8.45 \mathrm{AU}$, an eccentricity $e_{12}=0.8$ and an orbital inclination of $i_{12}=102.9^{\circ}$, and merge within $\sim 1.5 \times 10^{7} \mathrm{yr}$.

an isotropic distribution (i.e. uniform in $\cos i$ ). The other relevant angles are drawn randomly.

After sampling the relevant parameters, we check that the initial configuration satisfies the stability criterion of hierarchical triples of Mardling \& Aarseth (2001), which is assumed to be valid for quadruple systems if the third companion is appropriately replaced by a binary system

$$
\frac{R_{p}}{a_{\text {in }}} \geqslant 2.8\left[\left(1+\frac{m_{\text {out }}}{m_{\text {in }, 1}+m_{\text {in }, 2}}\right) \frac{1+e_{\text {out }}}{\sqrt{1-e_{\text {out }}}}\right]^{2 / 5} .
$$

In the previous equation, $m_{\mathrm{in}, 1}$ and $m_{\mathrm{in}, 2}$ represent the masses of the inner binary, $m_{\text {out }}$ the total mass of the binary companion and $R_{p}=a_{\text {out }}\left(1-e_{\text {out }}\right)$ is the pericentre distance of the outer orbit.

Given the above set of initial parameters, we integrate the system of differential equations of motion of the 4-bodies

$$
\ddot{\mathbf{r}}_{i}=-G \sum_{j \neq i} \frac{m_{j}\left(\mathbf{r}_{i}-\mathbf{r}_{j}\right)}{\left|\mathbf{r}_{i}-\mathbf{r}_{j}\right|^{3}}
$$



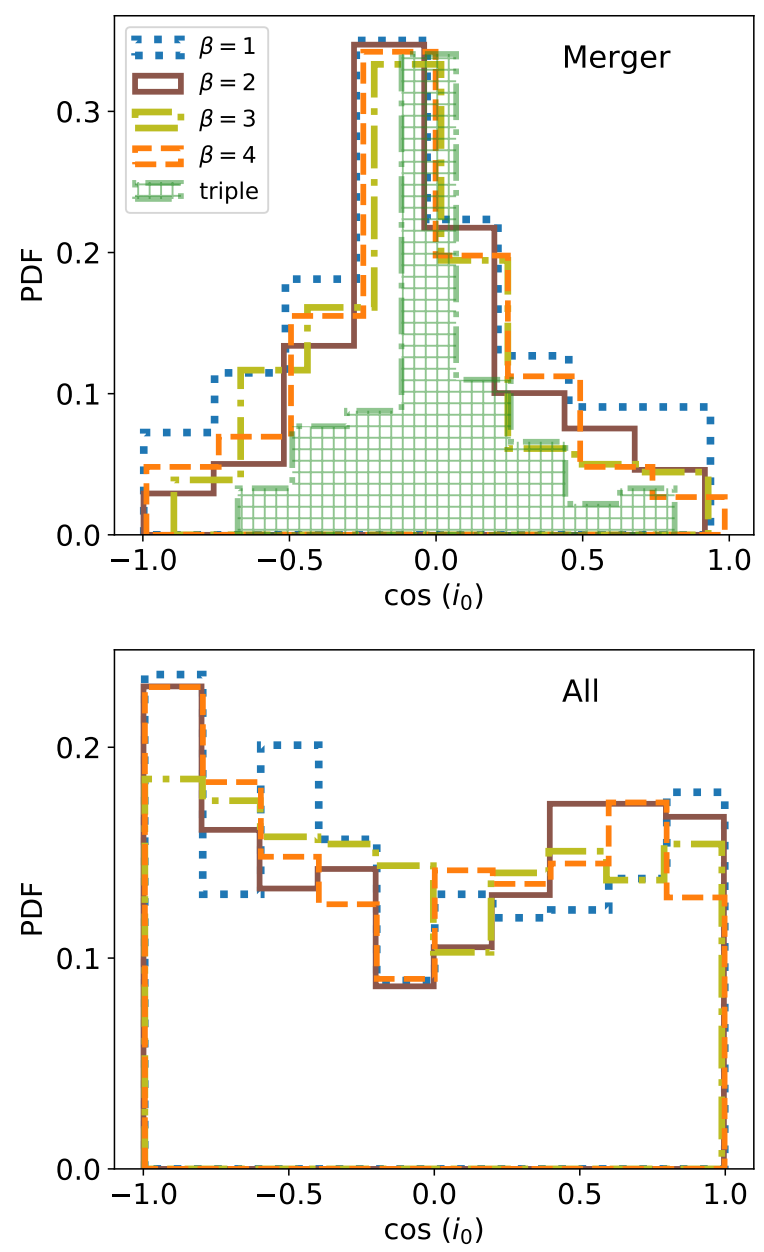

Figure 3. Initial inclination distribution of $\mathrm{BH}$ binaries that merge (top) and that do not merge in the simulation (bottom). Compared to triple systems, excursions to very high eccentricity can take place over a much larger fraction of the parameter space and the distribution is broader.

with $i=1,2,3,4$, by means of the ARCHAIN code (Mikkola \& Merritt 2006, 2008), a fully regularized code able to model the evolution of binaries of arbitrary mass ratios and eccentricities with high accuracy and that includes PN corrections up to order PN2.5. We performed 1000 simulations for each model in Tab. 1. We fix the maximum integration time as (Silsbee \& Tremaine 2017)

$$
T=\min \left(10^{3} \times \max \left(T_{\mathrm{LK}, 12}, T_{\mathrm{LK}, 34}\right), 10 \mathrm{Gyr}\right),
$$

where $T_{\mathrm{LK}, 12}$ and $T_{\mathrm{LK}, 34}$ are the LK timescales of the first $\left(m_{1}, m_{2}\right)$ and second $\left(m_{3}, m_{4}\right)$ inner binary, respectively.

Figure 2 shows the results of a four-body integration of one representative quadruple system with masses $m_{1}=$ $5.4 \mathrm{M}_{\odot}, m_{2}=7.5 \mathrm{M}_{\odot}, m_{3}=9.1 \mathrm{M}_{\odot}, m_{4}=9.4 \mathrm{M}_{\odot}$. The first two BHs start with an orbital inclination of $i_{12}=102.9^{\circ}$ with respect to the quadruple orbital plane, semi-major axis $a_{12}=8.45 \mathrm{AU}$, and eccentricity $e_{12}=0.8$. As a consequence of the efficient dissipation at high eccentricities pumped up by the LK effect (bottom panel), the BHs merge after $1.5 \times 10^{7} \mathrm{yr}$. We note that binaries may enter the non-secular regime during the maximum of an LK oscillation and the secular equation of motions are a poor description of the
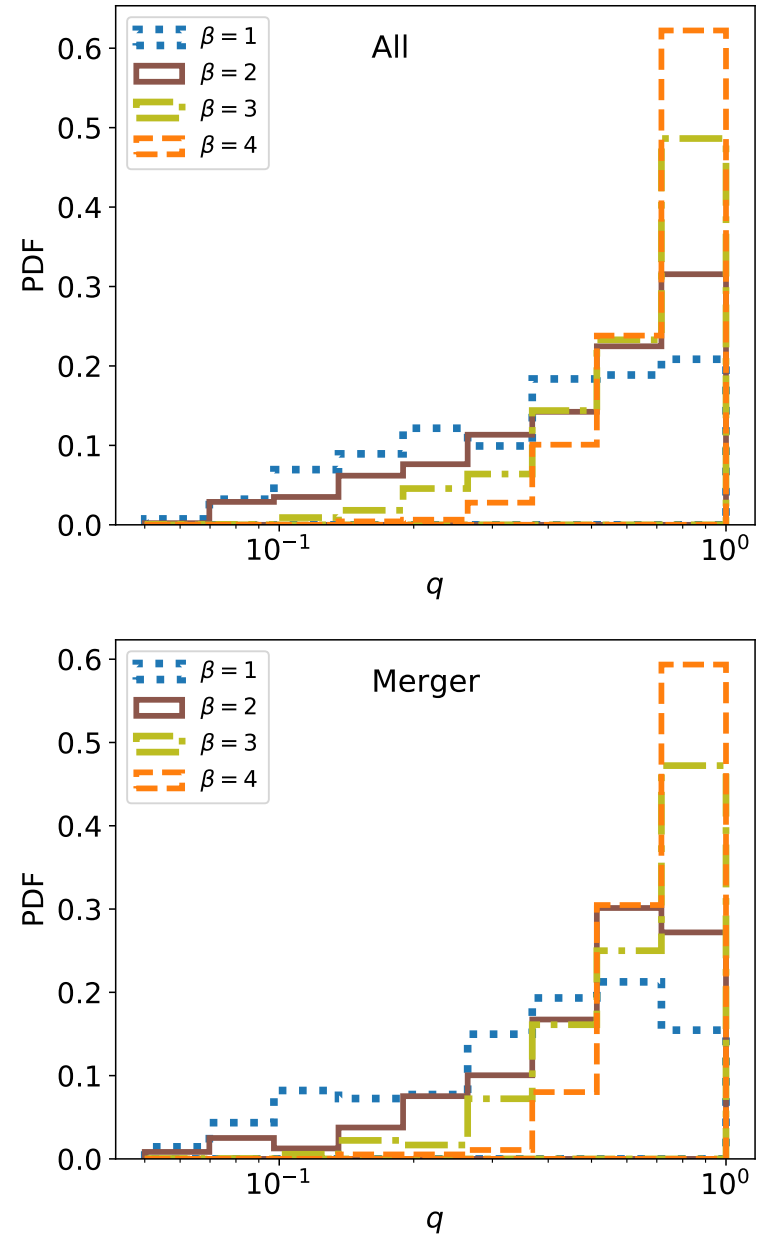

Figure 4. Mass ratio distribution of $\mathrm{BH}$ binaries in quadruples in the simulation (top) and of $\mathrm{BH}$ binaries that merge (bottom) for different values of $\beta$. There is no significant enhancement of merger probability for any mass ratio in comparison to the prior mass ratio distribution.

quadruple dynamics. In this regime, the inner binary BHs can be driven to a merger before general relativistic effects suppress the LK oscillations.

We now describe the key physical properties of the BHs that merge in our models.

\subsection{Inclination distribution}

In Figure 3, we show the probability distribution function (PDF) of the initial inclination between the inner binary orbit and the outer orbit of the $\mathrm{BH}$ binaries in quadruples that lead to a merger (top panel) and do not lead to a merger (bottom panel), for different slopes $\beta$ of the $\mathrm{BH}$ mass function (Models A). Figure 3 shows that the majority of the merging systems have initially high inclinations and that the distributions peak at $\sim 90^{\circ}$. However, binaries in quadruples can experience coherent eccentricity oscillations and excursions to very high eccentricity over a much larger fraction of the parameter space compared to triple systems (Pejcha et al. 2013) and possible resonances between the nodal precessions and the LK oscillations can arise (Hamers \& Lai 2017). As a consequence, the inclination distribution 

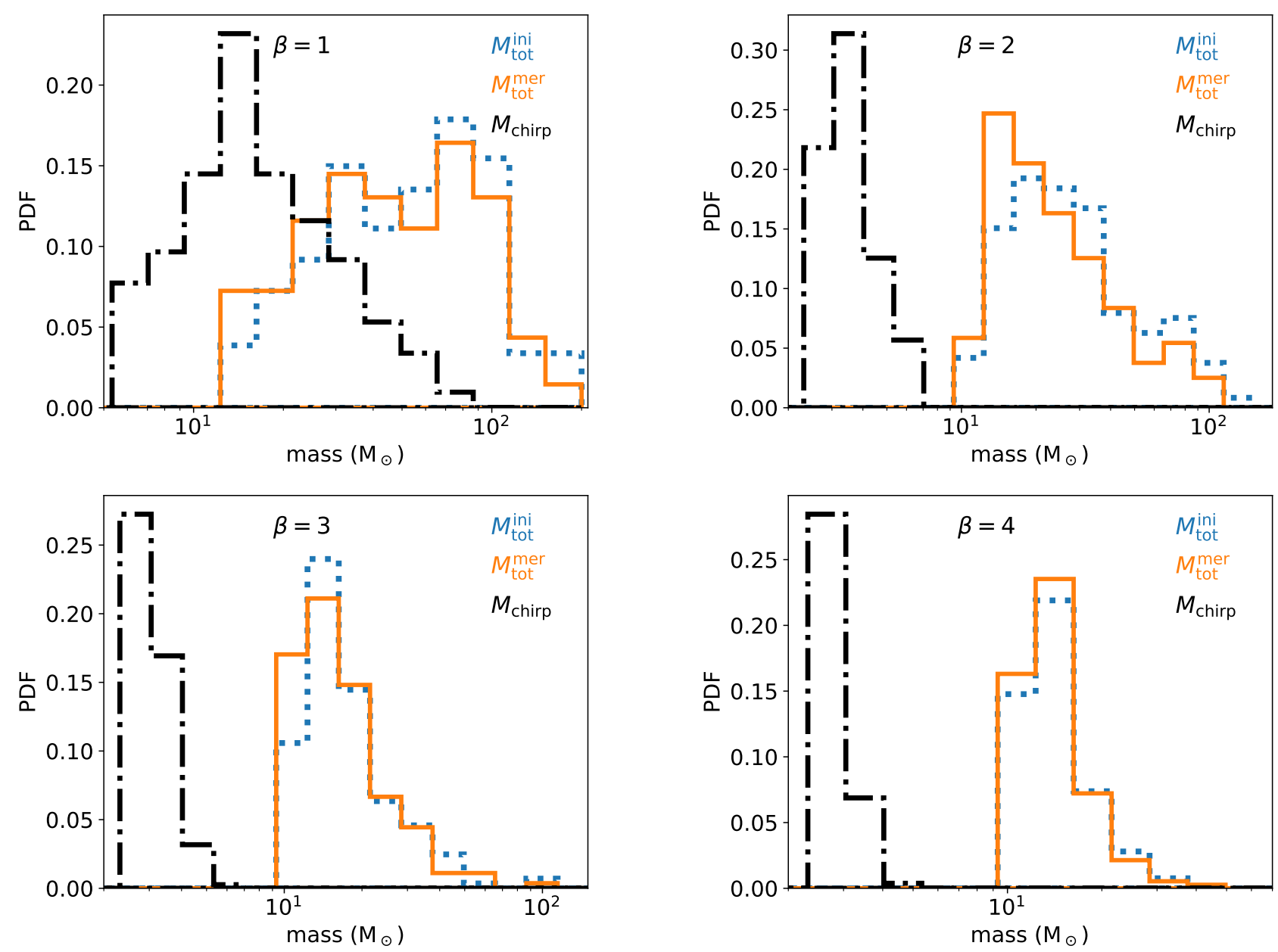

Figure 5. Total mass distribution of $\mathrm{BH}$ binaries in quadruples, total mass of $\mathrm{BH}$ binaries that lead to a merger, and chirp mass for $\beta=1$ (top-left), $\beta=2$ (top-right), $\beta=3$ (bottom-left), $\beta=4$ (bottom-right).

of merging BHs in our four-body simulations shows broader tails compared to the BH mergers in triples. To show this, we have run an additional model where we replace in Model A1 one of the binaries with a single $\mathrm{BH}$ of equivalent total mass $^{1}$. Figure 3 shows that the distribution still peaks at $\sim 90^{\circ}$, but with less important tails compared to the quadruple case. In particular, we find that $\sim 50 \%$ of the mergers in the triple case have initial inclination in the range $80^{\circ}-100^{\circ}$, while this fraction decreases to $\sim 30 \%$ for a quadruple $\mathrm{BH}$.

\subsection{Masses}

To examine the expected total mass $m_{\text {tot }}$ and the chirp mass

$$
M_{\text {chirp }}=m_{\text {tot }} \frac{q^{3 / 5}}{(1+q)^{6 / 5}}
$$

of the merging binaries in quadruples $\left(q=m_{2} / m_{1}\right.$, with $m_{1}>m_{2}$, is the mass ratio of merging $\mathrm{BHs}$ ), we have run models with different values of the slope of the $\mathrm{BH}$ mass function.

\footnotetext{
1 In these runs, we adopt as maximum integration time $T=$ $\min \left(10^{3} \times T_{\mathrm{LK}, 12}, 10 \mathrm{Gyr}\right)$, where $T_{\mathrm{LK}, 12}$ is the LK timescale of the inner binary of the triple.
}

Figure 4 reports the distribution of mass ratios of $\mathrm{BH}$ binaries in quadruples (top) and of $\mathrm{BH}$ binaries in quadruples (bottom) that lead to a merger for different $\beta$ 's. We find that the distribution of mass ratios of merging $\mathrm{BH}$ binaries maps the overall distribution for all the values of $\beta$. Steeper mass functions predict mergers of BHs with similar masses, while shallower mass functions allow a broader distribution with mass ratio down to $q \sim 0.05$. The mass ratio distribution of merging systems follows the prior mass ratio distribution of randomly drawing two objects from the $\mathrm{BH}$ mass function.

Figure 5 reports the total mass distribution of $\mathrm{BH}$ binaries in quadruples, total mass of $\mathrm{BH}$ binaries that lead to a merger, and chirp mass for different $\beta$ 's. Our merging binaries have total masses in the range $\sim 10-110 \mathrm{M}_{\odot}$, with the shape of the distribution that depends on the assumed slope of the BH mass function. Steeper mass functions (i.e. larger $\beta$ 's) result in a smaller average $m_{\text {tot }}$. We find that the total mass distribution of all binaries (including those which do not merge) is roughly constant for $\gtrsim 20 \mathrm{M}_{\odot}$ for $\beta=1$, while it is peaked at smaller total masses for larger $\beta$, i.e. at $\sim 20 \mathrm{M}_{\odot}$ for $\beta=2$ and $\sim 15 \mathrm{M}_{\odot}$ for $\beta=3-4$. We find that $\sim 90 \%$ of the mergers have total mass $\lesssim 50 \mathrm{M}_{\odot}, \lesssim 30 \mathrm{M}_{\odot}$, and $\lesssim 20 \mathrm{M}_{\odot}$ for $\beta=2,3$, and 4 , respectively. For all $\beta$ 's, we 

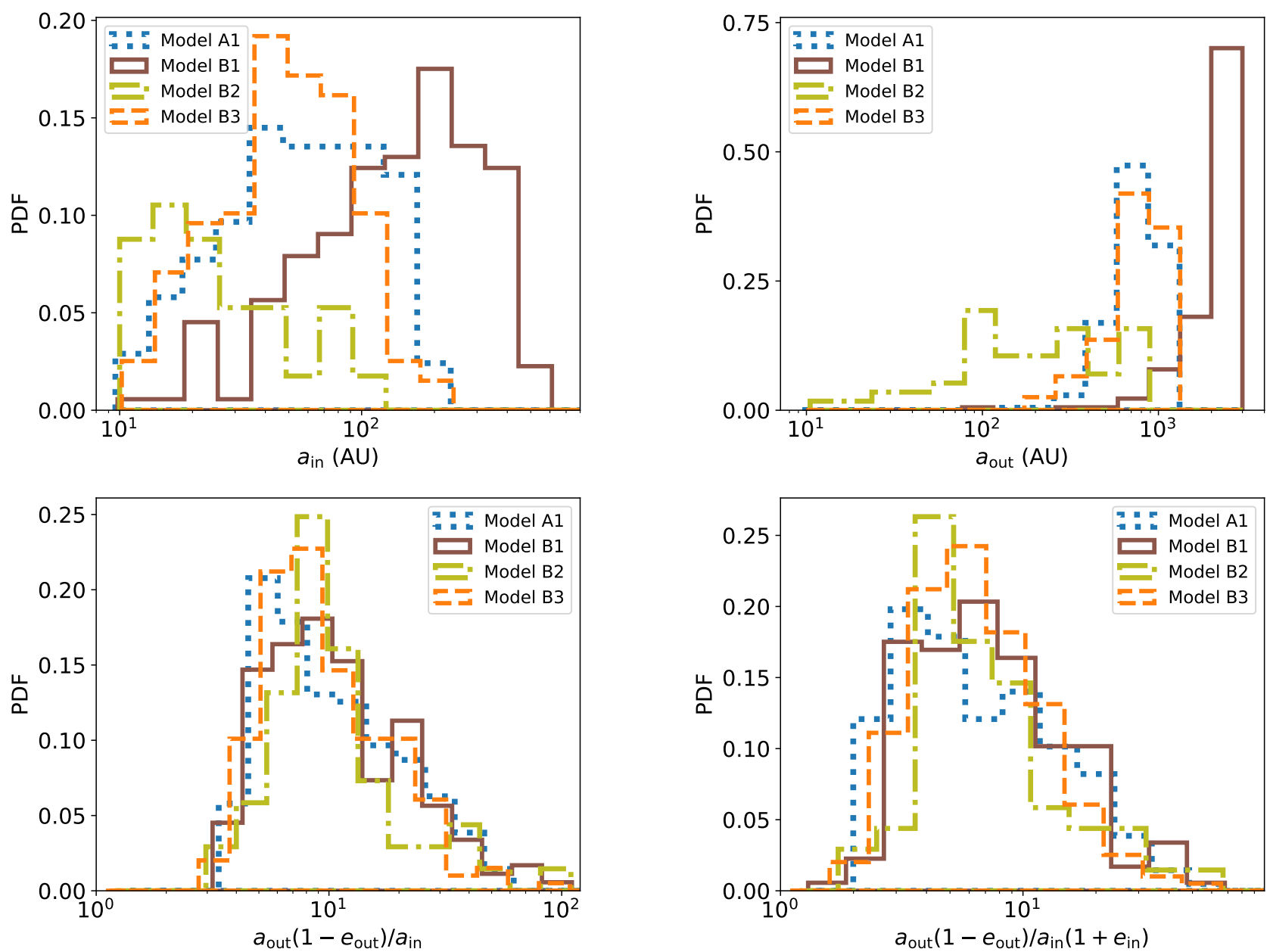

Figure 6. Orbital distributions of of BH binaries in quadruples that lead to a merger for Models A1 and B (see Tab. 1): inner semi-major axis (top-left), inner eccentricity (top-right), outer semi-major axis (bottom-left), ratio of the outer pericentre to the inner semi-major axis (bottom-right).

find that the distribution of the total mass of merging $\mathrm{BH}$ binaries tracks the underlying total mass distribution. Thus, there is no significant enhancement of the merger probability for any value of the total mass with respect to the prior distribution.

The distribution of chirp masses also depends on $\beta$, but the dependence is less sharp than the total mass. In the case $\beta=1$, we find that $M_{\text {chirp }}$ is in the range $\sim 5-100 \mathrm{M}_{\odot}$ and its distribution presents a peak at $\sim 15 \mathrm{M}_{\odot}$. The peak of the distribution is at $\sim 3 \mathrm{M}_{\odot}$ and $M_{\text {chirp }}$ is in the range $\sim 3-7 \mathrm{M}_{\odot}$ for $\beta=2$. Larger values of $\beta$ do not affect significantly neither the peak nor the shape of the chirp mass distribution.

\subsection{Orbital semi-major axis and eccentricity}

Figure 6 illustrates the distributions of orbital elements of the $\mathrm{BH}$ binaries in quadruples that lead to a merger for Model B.

In the top panel, we illustrate the distributions of inner (left) and outer (right) semi-major axes of merging binaries. The shape of the $a_{\text {in }}$ and $a_{\text {out }}$ distributions are mainly set by the initial distribution of semi-major axes and by the maximum extent of the quadruple $a_{\max }^{\text {out }}$. In the case of Model $\mathrm{A} 1\left(a_{\max }^{\text {out }}=1000 \mathrm{AU}\right.$ and $f(e)$ uniform $)$, the distribution of inner semi-major axes is peaked at $\sim 50 \mathrm{AU}$ and extends up to $\sim 200 \mathrm{AU}$. The distribution shifts to larger values with a peak at $200 \mathrm{AU}$ in Model B2, where the initial maximum semi-major axes was set to $3000 \mathrm{AU}$, while it is peaked at $\sim 15 \mathrm{AU}$ in Model B2, where the initial semi-major axes are drawn from a log-uniform distribution. A similar trend is present in the distributions of outer semi-major axis, which are peaked at $\sim 900 \mathrm{AU}, 2500 \mathrm{AU}, 100 \mathrm{AU}$ for Model A1, Model B1 and Model B2, respectively.

The adopted distribution of initial eccentricities, i.e. either uniform (Model A1) or thermal (Model B3), does not affect the distributions of inner and outer semi-major axis of merging systems.

In the bottom panel of Figure 6, we plot the distribution of the ratio of the outer pericentre to the inner semi-major axis (left) and the distribution of the ratio of the outer pericentre to the inner apocentre (right) for merging systems. Both distributions are almost independent of the initial distribution of orbital semi-major axes and eccentricities. We find that the distributions peak at $a_{\text {out }}\left(1-e_{\text {out }}\right) / a_{\text {in }} \sim 10$ 

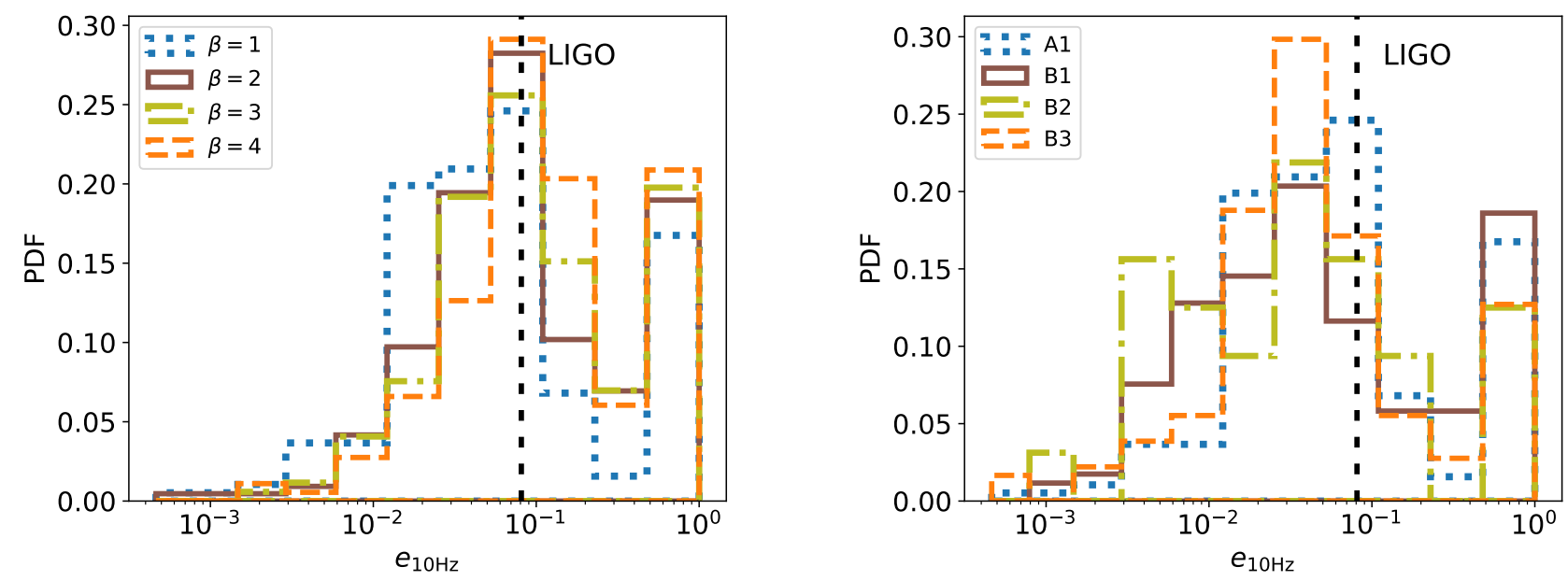

Figure 7. Distribution of eccentricities at the moment the BH binaries enter the LIGO frequency band (10 Hz) for mergers produced by quadruples. Left panel: Model A; right panel: Models A1 and B (see Tab. 1). The vertical line shows the minimum $e_{10 \mathrm{~Hz}}=0.081$ where LIGO/VIRGO/KAGRA network may distinguish eccentric sources from circular sources (Gondán \& Kocsis 2019). A significant fraction of binaries formed in quadruples have a significant eccentricity in the LIGO band.

and $a_{\text {out }}\left(1-e_{\text {out }}\right) /\left[a_{\text {in }}\left(1+e_{\text {in }}\right)\right] \sim 5$ for merging binaries in quadruple systems universally for all of our models.

\subsection{Eccentricity in the LIGO band}

Figure 7 shows the distribution of eccentricities at the moment the peak $\mathrm{GW}$ frequency of the $\mathrm{BH}$ binaries enter the LIGO frequency band $\left(f_{\mathrm{GW}}=10 \mathrm{~Hz}\right)$ for mergers produced by quadruples, both for Model A (left) and Model B (right). Eccentric binaries emit a GW signal with a broad spectrum of frequencies. We compute the peak frequency of the GW spectrum as (Wen 2003)

$$
f_{\mathrm{GW}}=\frac{\sqrt{G\left(m_{1}+m_{2}\right)}}{\pi} \frac{\left(1+e_{\mathrm{in}}\right)^{1.1954}}{\left[a_{\mathrm{in}}\left(1-e_{\mathrm{in}}^{2}\right)\right]^{1.5}} .
$$

Figure 7 shows that binaries formed in quadruples have larger eccentricities than those formed through many other channels, particularly mergers in isolated binary evolution and in SBHB ejected from star clusters (Fragione \& Kocsis 2018; Giacobbo \& Mapelli 2018; Rodriguez et al. 2018). However, mergers that follow from the GW capture scenario in clusters (Zevin et al. 2019) and galactic nuclei (O'Leary et al. 2009; Gondán et al. 2018; Rasskazov \& Kocsis 2019), from resonant binary-single scattering in clusters (Samsing et al. 2018b), from hierarchical triples (Antonini et al. 2017; Fragione et al. 2018, 2019; Fragione \& Loeb 2019), and from $\mathrm{BH}$ binaries orbiting intermediate-mass black holes in star clusters (Fragione \& Bromberg 2019) also present a similar peak at high eccentricities. We note that the high eccentricities we find in the binaries that merge in our simulations may imply that a fraction of these binaries could emit their maximum power at higher frequencies, possibly in the range of LISA. However, binaries that enter the LIGO band with high eccentricities $\left(\gtrsim 10^{-3}\right)$ could be harder to detect with instruments like LISA (Chen \& Amaro-Seoane 2017). Nevertheless, Randall \& Xianyu (2019) and Hoang et al. (2019) have proposed that LK oscillations of binary BHs in a triple configuration have the potential to be observed by LISA. In

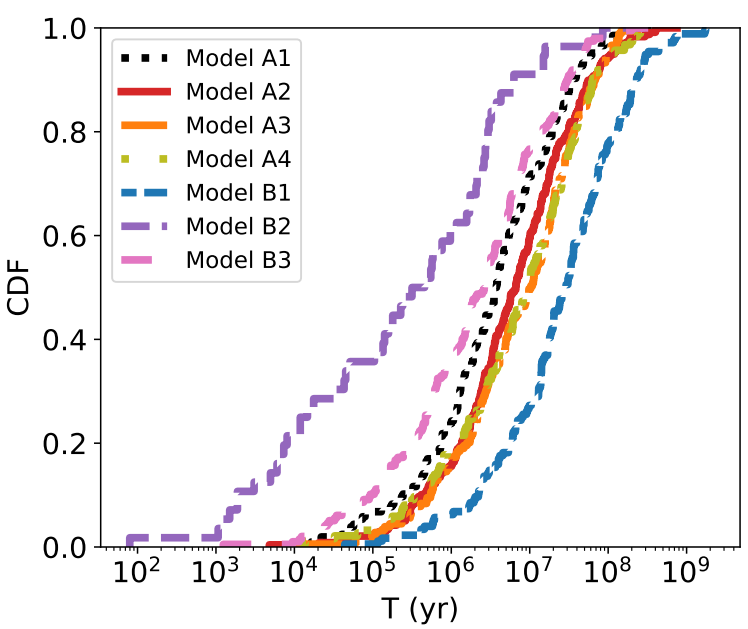

Figure 8. Merger time distribution of $\mathrm{BH}$ binaries in quadruples that lead to merger for all models (see Tab.1).

the quadruple case, both the inner $\mathrm{BH}$ binaries can enter the LISA frequency band and produce observable eccentricity variations if their respective LK cycles happen to have similar duration.

\subsection{Merger times}

Figure 8 shows the merger time cumulative distribution functions (CDFs) of $\mathrm{BH}$ binaries in quadruples that lead to merger for Model A and Model B. The CDF function depends both on the maximum extent of the quadruple $a_{\max }^{\text {out }}$ and on the initial distribution of semi-major axes, but it does not depend on the assumed slope of the $\mathrm{BH}$ mass function nor on the initial distribution of eccentricities. Larger $a_{\max }^{\text {out }}$ imply a larger merger timescale. In Model A1 ( $a_{\max }^{\text {out }}=1000$ $\mathrm{AU})$, we find that $\sim 50 \%$ of the mergers happen within $\sim 10^{7} \mathrm{yr}$, while in Model B1 $\left(a_{\max }^{\text {out }}=3000 \mathrm{AU}\right)$ within 
$\sim 10^{8}$ yr. In Model B2, where we sample the initial semimajor axes from a log-uniform distribution, $\sim 50 \%$ of the BHs merge within $\sim 10^{6}$ yr.

\section{DISCUSSION AND CONCLUSIONS}

In this paper, we have studied the dynamical evolution of a bound $2+2$ type quadruple $\mathrm{BH}$ to estimate if this channel can contribute to the observed rate of BHs mergers. As found in Liu \& Lai (2019), quadruples have one additional degree of freedom and a richer dynamics, which can enhance the merger fraction.

We carried out a systematic statistical study of these systems by means of high-precision direct $N$-body simulations including Post-Newtonian (PN) terms up to $2.5 \mathrm{PN}$ order. We ran a series of simulations sampling a power-law mass spectrum for the BHs, different maximum extensions of the quadruple and different distributions of the inner and outer semi-major axes and eccentricities (Table 1).

- We found that the majority of the merging systems have high initial inclinations and that the distributions peak at $\sim 90^{\circ}$, as for triples, but with a broader distribution tail.

- We have also shown that the mass distribution of merging $\mathrm{BH}$ binaries simply follows the assumed prior distribution, thus there is no significant enhancement of the merger probability as a function of total mass, chirp mass, or mass ratio. The slope of the $\mathrm{BH}$ mass function affects the total mass distribution and the chirp mass distribution: the steeper the mass function, the smaller the average $m_{\text {tot }}$ and $M_{\text {chirp }}$. Specifically, $\sim 90 \%$ of the mergers have total mass $\lesssim 50 \mathrm{M}_{\odot}, \lesssim 30 \mathrm{M}_{\odot}, \lesssim 20 \mathrm{M}_{\odot}$ for $\beta=2, \beta=3, \beta=4$, respectively, assuming that the $\mathrm{BH}$ mass function extends to $100 \mathrm{M}_{\odot}$.

- The shape of the inner and outer semi-major axes distributions of merging systems, as well as the typical merging timescale, are mainly set by the initial distribution of semi-major axes and by the maximum extent of the quadruple $a_{\max }^{\text {out }}$. The initial eccentricity distribution of the inner binaries does not play a significant role. We find that the distributions peak at $a_{\text {out }}\left(1-e_{\text {out }}\right) / a_{\text {in }} \sim 10$ and $a_{\text {out }}\left(1-e_{\text {out }}\right) /\left[a_{\text {in }}\left(1+e_{\text {in }}\right)\right] \sim 5$ for merging binaries in quadruple systems universally for all of our models.

- Finally, we have computed the eccentricity in the LIGO band and showed that BHs merging through this channel generate significant eccentricities. These are typically larger than BHs formed through mergers in isolated binary evolution and in SBHB ejected from star clusters (Fragione \& Kocsis 2018; Giacobbo \& Mapelli 2018; Rodriguez et al. 2018), but comparable to mergers that follow from the GW capture scenario in clusters (Samsing et al. 2018b; Zevin et al. 2019) and galactic nuclei (O'Leary et al. 2009; Gondán et al. 2018; Rasskazov \& Kocsis 2019), from hierarchical triples (Antonini et al. 2017), and from BH binaries orbiting intermediate-mass black holes in star clusters (Fragione \& Bromberg 2019). Correlations between mass and eccentricity may distinguish some of these merger channels (Gondán et al. 2018).

To directly compare to triples, we have also run an additional model where we replace in Model A1 one of the binaries with a single $\mathrm{BH}$ of equivalent total mass. We have found that the distribution of inclinations of $\mathrm{BH}$ binaries that lead to a merger is still peaked at $\sim 90^{\circ}$. However, $\sim 50 \%$ of the mergers in the triple case have initial inclination in the range $80^{\circ}-100^{\circ}$, while this fraction decreases to $\sim 30 \%$ for a quadruple BH. The distributions of masses, mass ratios and orbital elements of the merging BHs are not affected if one of the binaries in a hierarchical quadruple is replaced with a single $\mathrm{BH}$ in our simulations. Finally, we also investigated the role of PN terms by running Model A1 without PN corrections to the equations of motion. We have found that the fraction of $\mathrm{BH}$ binaries that merge decreases drastically. The reason is that there is no efficient energy dissipation due to GW emission at the pericenter when the eccentricity is excited as a consequence of the LK mechanism. The only available path for a merger is a head-on collision, when the two BHs reach a pericentre smaller than the sum of their Schwarzschild radii, typically of the order $\sim 10^{-7}$ AU. This means that, if the inner semi-major axis is $\sim 10 \mathrm{AU}$, the LK mechanism should excite the eccentricity to $1-e_{\max } \sim 10^{-8}$, hence the initial inclination should be in the tiny interval $\sim 89.996-90.004$.

To accurately determine the global $\mathrm{BH}$ merger rate from quadruples, we would need to quantify the population of massive quadruple stars that lead to a quadruple $\mathrm{BH}$ of a given mass, which is currently poorly known. In our study, we have assumed a simple power-law distribution of $\mathrm{BH}$ masses in the range $5 \mathrm{M}_{\odot}-100 \mathrm{M}_{\odot} \cdot{ }^{2}$ The multiplicity fraction of high-mass main-sequence stars, which may be the progenitors of NSs and BHs, can be as high as $\sim 80 \%$ and quadruples are not rare, representing $\sim 20 \%-25 \%$ of the fraction of stellar objects in triples (Sana 2017). We find that the merger fraction in quadruples can be larger than that for triples, even up to $\sim 3-4$ times larger (see the last column of Tab. 1). Our results thus suggest that dynamically-driven $\mathrm{BH}$ and NS mergers in the quadruple scenario can be an important contribution to the events observed by LIGO/VIRGO.

\section{ACKNOWLEDGEMENTS}

We thank Dong Lai for useful comments. GF thanks Seppo Mikkola for helpful discussions on the use of the code ARCHAIN. GF is supported by the Foreign Postdoctoral Fellowship Program of the Israel Academy of Sciences and Humanities. GF also acknowledges support from an Arskin postdoctoral fellowship at the Hebrew University of Jerusalem. GF acknowledges hospitality from the Eötvös Loránd University of Budapest. This project has received funding from the European Research Council (ERC) under the European Union's Horizon 2020 research and innovation programme under grant agreement No 638435 (GalNUC) and by the Hungarian National Research, Development, and Innovation Office grant NKFIH KH-125675 (to BK). Simulations were run on the Astric cluster at the Hebrew University of Jerusalem.
2 Note that pulsational pair instability may limit the maximum mass to $\sim 50 \mathrm{M}_{\odot}($ Belczynski et al. 2016). 


\section{REFERENCES}

Antognini J. M. O., 2015, MNRAS, 452, 3610

Antonini F., Chatterjee S., Rodriguez C. L., Morscher M., Pattabiraman B., Kalogera V., Rasio F. A., 2016, ApJ, 816,65

Antonini F., Perets H. B., 2012, ApJ, 757, 27

Antonini F., Toonen S., Hamers A. S., 2017, ApJ, 841, 77

Arca-Sedda M., Li G., Kocsis B., 2018, arXiv e-prints

Askar A., Szkudlarek M., Gondek-Rosińska D., Giersz M., Bulik T., 2017, MNRAS, 464, L36

Banerjee S., 2018, MNRAS, 473, 909

Bartos I., Kocsis B., Haiman Z., Márka S., 2017, ApJ, 835, 165

Belczynski K., Heger A., Gladysz W., Ruiter A. J., Woosley S., Wiktorowicz G., Chen H. Y., Bulik T., O'Shaughnessy R., Holz D. E., Fryer C. L., Berti E., 2016, A\& A, 594, A97

Belczynski K., Holz D. E., Bulik T., O'Shaughnessy R., 2016, Nature, 534, 512

Bird S., Cholis I., Muñoz J. B., Ali-Haïmoud Y., Kamionkowski M., Kovetz E. D., Raccanelli A., Riess A. G., 2016, Physical Review Letters, 116, 201301

Breiter S., Vokrouhlický D., 2018, MNRAS, 475, 5215

Chen X., Amaro-Seoane P., 2017, ApJL, 842, L2

Choksi N., Volonteri M., Colpi M., Gnedin O. Y., Li H., 2018, arXiv:1809.01164

Dunstall P. R., Dufton P. L., Sana H., Evans C. J., Howarth I. D., Simón-Díaz S., de Mink S. E., Langer N., Maíz Apellániz J., Taylor W. D., 2015, A\& A, 580, A93

Duquennoy A., Mayor M., 1991, A\& A, 248, 485

Fang X., Thompson T. A., Hirata C. M., 2017, arXiv

Fragione G., Bromberg O., 2019, arXiv e-prints, p. arXiv:1903.09659

Fragione G., Grishin E., Leigh N. W. C., Perets H. B., Perna R., 2018, arXiv e-prints

Fragione G., Kocsis B., 2018, Phys Rev Lett, 121, 161103

Fragione G., Leigh N., Perna R., 2019, arXiv e-prints, p. arXiv:1903.09160

Fragione G., Loeb A., 2019, arXiv e-prints, p. arXiv:1903.10511

Giacobbo N., Mapelli M., 2018, MNRAS, 480, 2011

Gondán L., Kocsis B., 2019, ApJ, 871, 178

Gondán L., Kocsis B., Raffai P., Frei Z., 2018, ApJ, 860, 5

Grishin E., Perets H. B., Fragione G., 2018, MNRAS, 481, 4907

Hamers A. S., 2018, MNRAS, 478, 620

Hamers A. S., Bar-Or B., Petrovich C., Antonini F., 2018, ApJ, 865, 2

Hamers A. S., Lai D., 2017, MNRAS, 470, 1657

Hoang B.-M., Naoz S., Kocsis B., Farr W., McIver J., 2019, arXiv e-prints

Hoang B.-M., Naoz S., Kocsis B., Rasio F. A., Dosopoulou F., 2018, ApJ, 856, 140

Jiménez-Esteban F. M., Solano E., Rodrigo C., 2019, AJ, 157, 78

Kozai Y., 1962, AJ, 67, 591

Li G., Naoz S., Kocsis B., Loeb A., 2014, ApJ, 785, 116

Lidov M. L., 1962, P\& SS, 9, 719

Liu B., Lai D., 2018, ApJ, 863, 68

Liu B., Lai D., 2019, MNRAS, 483, 4060

Mandel I., de Mink S. E., 2016, MNRAS, 458, 2634
Marchant P., Langer N., Podsiadlowski P., Tauris T. M., Moriya T. J., 2016, A\& A, 588, A50

Mardling R. A., Aarseth S. J., 2001, MNRAS, 321, 398

Michaely E., Perets H. B., 2019, MNRAS, 484, 4711

Mikkola S., Merritt D., 2006, MNRAS, 372, 219

Mikkola S., Merritt D., 2008, AJ, 135, 2398

Naoz S., 2016, ARA\& A, 54, 441

Naoz S., Farr W. M., Lithwick Y., Rasio F. A., Teyssandier J., 2013, MNRAS, 431, 2155

Naoz S., Kocsis B., Loeb A., Yunes N., 2013, ApJ, 773, 187

O'Leary R. M., Kocsis B., Loeb A., 2009, MNRAS, 395, 2127

O'Leary R. M., Meiron Y., Kocsis B., 2016, ApJLett, 824, L12

O'Leary R. M., Rasio F. A., Fregeau J. M., Ivanova N., O'Shaughnessy R., 2006, ApJ, 637, 937

Pejcha O., Antognini J. M., Shappee B. J., Thompson T. A., 2013, MNRAS, 435, 943

Petrovich C., Antonini F., 2017, ApJ, 846, 146

Portegies Zwart S. F., McMillan S. L. W., 2000, ApJL, 528, L17

Raghavan D., et al., 2010, ApJSuppl, 190, 1

Randall L., Xianyu Z.-Z., 2019, arXiv e-prints, p. arXiv:1902.08604

Rasskazov A., Kocsis B., 2019, arXiv e-prints, p. arXiv:1902.03242

Rastello S., Amaro-Seoane P., Arca-Sedda M., CapuzzoDolcetta R., Fragione G., Tosta e Melo I., 2018, MNRAS

Riddle R. L., Tokovinin A., Mason B. D., Hartkopf W. I., Roberts Jr. L. C., Baranec C., Law N. M., Bui K., Burse M. P., Das H. K., Dekany R. G., Kulkarni S., Punnadi S., Ramaprakash A. N., Tendulkar S. P., 2015, ApJ, 799, 4

Rodriguez C. L., Amaro-Seoane P., Chatterjee S., Rasio F. A., 2018, PRL, 120, 151101

Samsing J., Askar A., Giersz M., 2018a, ApJ, 855, 124

Samsing J., Askar A., Giersz M., 2018b, ApJ, 855, 124

Sana H., 2017, in Eldridge J. J., Bray J. C., McClelland L. A. S., Xiao L., eds, The Lives and Death-Throes of Massive Stars Vol. 329 of IAU Symposium, The multiplicity of massive stars: a 2016 view. pp 110-117

Sana H., et al., 2013, A\& A, 550, A107

Sasaki M., Suyama T., Tanaka T., Yokoyama S., 2016, Physical Review Letters, 117, 061101

Seto N., 2018, MNRAS, 475, 1392

Silsbee K., Tremaine S., 2017, ApJ, 836, 39

Stone N. C., Metzger B. D., Haiman Z., 2017, mnras, 464, 946

Tagawa H., Kocsis B., Saitoh T. R., 2018, ArXiv e-prints The LIGO Scientific Collaboration the Virgo Collaboration 2018, arXiv e-prints, p. arXiv:1811.12907

Tokovinin A., 2014a, AJ, 147, 86

Tokovinin A., 2014b, AJ, 147, 87

Vokrouhlický D., 2016, MNRAS, 461, 3964

Wen L., 2003, ApJ, 598, 419

Zevin M., Samsing J., Rodriguez C., Haster C.-J., RamirezRuiz E., 2019, ApJ, 871, 91 